\title{
Immuntherapie in der Uro-Onkologie II
}

\author{
Essen, 7. -8. Oktober 1994
}

\author{
W. K. Brenner, F. Steinbach \\ Urologische Klinik und Poliklinik, Klinikum der Johannes Gutenberg-Universität Mainz
}

Nach einem ersten Symposium zu diesem Thema vor drei Jahren fand die zweite Veranstaltung über Immuntherapie in der Uro-Onkologie unter der Leitung von Herrn Prof. Dr. Rübben am 7. und 8. Oktober in Essen statt. Insgesamt erfreute sich diese Veranstaltung aufgrund der hohen Aktualität der Problematik einer guten Besucherzahl sowie angeregten Diskussionen zu den vorgestellten Beiträgen. Das Symposium gliederte sich in zwei Abschnitte: Zunächst wurden experimentelle Ansätze zur Immuntherapie urologischer Tumoren und daran anschlieBend bereits vorhandene klinische Erfahrungen diskutiert.

\section{Experimentelle Ansätze zur Immun- therapie urologischer Tumoren}

Von $R$. J. A. van Moorselaar aus Nijmwegen wurden molekularbiologische Grundlagen der Gentherapie erläutert. Dabei wurde besonders die Problematik der zum gegenwärtigen Zeitpunkt am häufigsten verwendeten Gentransfermethode mittels Retroviren aufgezeigt. Die hohe Effizienz der Transduktion genetischen Materials ist bei diesem Vektorsystem mit dem Nachteil der undefinierten Lokalisation des eingebauten genetischen Materials verbunden. Eine Aktivierung von Onkogenen kann somit nicht sicher ausgeschlossen werden. Zudem erfolgt die Transduktion nur in sich teilende Zellen. In Zukunft soll daher der Einsatz anderer Vektorsysteme, z. B. von Adenoviren, geprüft werden. In dem Zusammenhang stellte T. Licht $(\mathrm{Be}-$ thesda, USA) die Möglichkeit einer Transfektion mit Liposomen vor, deren klinischer Einsatz noch geprüft werden muß.

G. Mickisch (Rotterdam) stellte erste konkrete Anwendungsbereiche der Gentherapie bei Patienten mit metastasiertem Urothelkarzinom der Harnblase vor. In dieser Klinik wird in Kürze eine Phase-I-Studie beginnen, in der bei entsprechend erkrankten Patienten das MDR1Gen in Knochenmarkstammzellen transduziert wird. Durch die anschließend erfolgende Expression des P-Glykoproteins werden diese Knochenmarkstammzellen chemotherapeutikaresistent und ermöglichen so eine deutliche Dosiseskalation. Als Chemotherapeutika werden Vinblastin und Doxorubicin eingesetzt.

Ein In-vitro-Testverfahren für neue Therapieverfahren wurde von $F$. Hofstädter (Regensburg) erläutert. Dieses System erlaubt ein dreidimensionales Tumorwachstum, indem sich ähnlich wie in vivo Tumorzellen un-

Akt. Urol. 26 (1995) 75-77

(C) Georg Thieme Verlag Stuttgart · New York terschiedlichen Differenzierungsgrades ausbilden. In der Regel werden dabei höhere Differenzierungsstufen der Tumorzellen im Vergleich zu den konventionellen zweidimensionalen Kultursystemen ausgebildet. Diese dreidimensionalen multizellulären Sphäroide erlauben das Studium von Zell-Zell-Interaktionen, Stroma-Tumorzell-Interaktionen sowie Untersuchungen zur Penetration von Antikörper und immunkompetenten Zellen in Tumoren.

In einem Übersichtsreferat stellte Th. Ebert (Düsseldorf) die Vor- und Nachteile verschiedener In-vivoTestverfahren dar. Hervorzuheben war neben dem Nacktmausmodell (T-Zell-Insuffizienz) die SCID-(Severe Combined Immune Deficiency)Maus, bei der sowohl ein Verlust der B- als auch T-Zellimmunität vorliegt. Dieser Immundefekt ist auf dem Chromosom 16 lokalisiert und wird autosomal rezessiv vererbt. Die SCID-Maus eignet sich besonders zur Erprobung antikörpervermittelter Immunstudien. Nachteilig ist die Gefahr der Graft-versus-Host-Reaktion bei der Xenotransplantation humaner peripherer Lymphozyten. Für diese Fälle ist die SCID-hu-Maus geeignet, die durch Transplantation humaner hämatopoetischer Stammzellen erzeugt wird. Die hierdurch generierten TZellen erkennen dann sowohl Human- als auch Mausantigene als eigen.

Prinzipien der Tumorinvasion und Metastasierung wurden am Beispiel des Harnblasenkarzinoms von T. Otto (Essen) erläutert. Verminderte E-Cadherin-Expression und gleichzeitig vermehrte Expression des humanen autokrinen Motilitätsfaktor-Rezeptors (hAMFR) gehen bei diesem Tumor mit einer Zunahme des Malignitätspotentials einher. Die Blockierung des hAMFR führt im Tiermodell zur Reduktion von Metastasen bei fortgeschrittenen Urothelkarzinomen der Harnblase.

In einem Referat von B. Schmitz-Dräger (Düsseldorf) zeigte sich, daß sich die Wirksamkeit einer Immuntherapie bis zum jetzigen Zeitpunkt am besten mit klinischen Parametern vorhersagen läßt. Hierzu gehören der Allgemeinzustand des Patienten, das Intervall zwischen Operation und Auftreten der Metastasen, die Metastasenlokalisation sowie bereits vorangegangene Chemo- und Immuntherapeutika. Mit serologischen und immunologischen Parametern ist es z.Z. nicht möglich, eine Patientenselektion pro oder kontra Immuntherapie vorzunehmen.

A. Harstrick (Essen) beschrieb urologische Indikationen zur Hochdosis-Chemotherapie, Stammzellseparation und Knochenmarktransplantation. Für diese Therapie sind aus dem urologischen Krankengut Patienten 
mit einem Hodentumorrezidiv sowie Blasenkarzinomrezidiv geeignet. Keine Indikationen stellen metastasierende Nierenzellkarzinome oder Prostatakarzinome dar. Bisherige klinische Studien ergaben, daß in der Rezidivsituation nach kompletter oder partieller Tumorremission nur eine Dosiserhöhung der bisher verwandten Chemotherapeutika, nicht jedoch alternative Chemotherapiekombinationen sinnvoll sind. Dabei bewirken sowohl die Stammzellseparation aus dem peripheren Blut sowie die in der Effektivität äquivalente Knochenmarkstransplantation eine Erhöhung der therapeutischen Breite und erlauben dadurch eine Dosissteigerung um das drei- bis fünffache. Voraussetzung für einen Erfolg dieser Therapie ist jedoch ein Ansprechen auf die Standardtherapie, dokumentiert z. B. durch einen Tumormarkerabfall beim Hodenkarzinom. Beim Ausbleiben jeglichen Ansprechens auf eine Standardtherapie besitzt der Patient auch keinen Vorteil von einer Hochdosistherapie. Bei rezidivierenden metastasierenden Hodentumoren konnte mit diesem Therapieverfahren eine 2-Jahres-Überlebensrate von $44 \%$ erzielt werden.

Molekulare und zytokinetische Aspekte der Entwicklung und Progression urologischer Tumoren wurden von $G$. Kovacs (Heidelberg) am Beispiel des Nierenzellkarzinoms dargestellt. Anhand spezifischer, zytokinetischer Veränderungen ist es im papillären Nierenkarzinom möglich, Adenome von malignen Nierenzellkarzinomen zu unterscheiden. Dabei sind Adenome im Gegensatz zu Karzinomen durch einen Verlust des Y-Chromosoms sowie eine Trisomie 7 und 17 gekennzeichnet. Beim nicht papillären Nierentumor ist die folgende genetische Konstellation mit einer erhöhten Metastasierungs-Wahrscheinlichkeit verbunden: $+5 q-6 q-8 p-9-14 q$. Aus der Kombination verschiedener genetischer Läsionen läßt sich somit eine Aussage über die Prognose des Patienten treffen.

\section{Klinische Immuntherapie urologischer Tumoren}

G. Nagel (Freiburg) diskutierte in seinem Vortrag den Stellenwert unkonventioneller Therapieverfahren bei der Behandlung maligner Tumoren. Danach haben alternative Therapiemethoden keinen direkten gegen den Tumor gerichteten zytokinetischen Effekt, bieten jedoch dem Patienten oft eine „Krücke“ im Sinne einer Überlebenshilfe.

In zwei Referaten wurden von Jung (Aachen) und Lamm (Morgantown, USA) die Erfahrungen der Chemo- und Immuntherapie des oberflächlichen Harnblasenkarzinoms in Europa sowie in den Vereinigten Staaten diskutiert. Unterschiedlich wurde in den Referaten der Effekt einer BCG-Instillationstherapie beim oberflächlichen Blasenkarzinom (Ta, T1) beurteilt. Während in der EORTC-Studie kein Unterschied zwischen den BCG- und Mitomycin-C-(MMC-)behandelten Patienten hinsichtlich der Rezidivrate war, hob sich in der von Lamm präsentierten Serie ein signifikanter Unterschied zugunsten der BCGbehandelten Patienten ab. Dabei zeigten Patienten mit einer BCG-Erhaltungs-Therapie, d.h. einer erneuten BCGInstillation 3 Monate nach dem ersten Instillationszyklus einen besseren Verlauf auf als Patienten, die ausschließlich initial behandelt wurden. Beim Carcinoma in situ konnte so die Rate der kompletten Remissionen von $73 \%$ bei einmaliger 6wöchiger Behandlung auf $87 \%$ gesteigert werden.

Von $G$. Riethmüller (München) wurde die Problematik des "minimal residual cancer" nach makroskopisch radikaler Tumorentfernung angesprochen, die in erster Linie in der Metastasierung liegt. Dabei sind Tumorzellen, z. B. $10^{7}-10^{8}$ einzelne Zellen, im mesenchymalen Gewebe der Lymphknoten für eine Therapie mit zytotoxischen monoklonalen Antikörpern gut zugänglich. Dies konnte am Beispiel des Kolonkarzinoms belegt werden, bei dem eine adjuvante Therapie von Patienten im Stadium D1 mit dem Antikörper 17-1A einen signifikanten Einfluß auf die Überlebensrate hatte, bei gleichzeitig geringer Nebenwirkungsrate. Der Einsatz monoklonaler Antikörper in der Therapie scheint daher eher in der Zerstörung mikroskopisch kleiner Tumorreste zu liegen und ist nicht als Alternativtherapieoption zur radikalen Primärtumoroperation zu sehen.

In mehreren Referaten wurde der derzeitige Stellenwert der Chemo- und Immuntherapie beim Nierenzellkarzinom aufgezeigt. Von $S$. Fossa (Oslo) wurde eine hochdosierte Interferon- $\alpha$-Therapie als das Therapieverfahren der Wahl beim metastasierten Nierenzellkarzinom angegeben. Jedoch konnte durch die alleinige Interferon- $\alpha$-Therapie nur eine 14\%ige Ansprechrate erzielt werden. In Kombination mit 5-FU oder Isotretinoin konnte diese auf 23 bw. $29 \%$ gesteigert werden. Kombinierte Chemo-/Immuntherapien sollten jedoch nur im Rahmen klinischer Studien durchgeführt werden.

E. Huland (Hamburg) präsentierte Ergebnisse der Inhalationsbehandlung mit Interleukin-2 beim metastasierenden Nierenzellkarzinom. Neben hoher Patientenakzeptanz ist diese Therapieform vor allem durch eine niedrige Nebenwirkungsrate gekennzeichnet. Aufgrund der geringen Toxizität sowie der einfachen technischen Handhabung des Inhalationsgerätes kann diese Therapie von den meisten Patienten unter häuslichen Bedingungen durchgeführt werden. Dabei wiesen von den ursprünglich behandelten Patienten $(n=18)$ nach einer durchschnittlichen Nachbeobachtungszeit von 18 Monaten und einer Behandlungsdauer von 13,6 Monaten ein Patient eine komplette Remission, 9 Patienten eine partielle Remission, 7 Patienten einen unveränderten Tumorstatus sowie 1 Patient ein progressives Tumorleiden auf.

Die Ergebnisse der systemischen Gabe von Interleukin-2 allein, bzw. in Kombination mit Interferon $\gamma$ und tumorinfiltrierenden Lymphozyten, wurde in einer "State of the art"-lecture von $A$. Belldegrun (Los Angeles) dargestellt. Auch in diesem Krankengut lag die Rate der kompletten Remissionen zwischen $1 \%$ und $6 \%$, die der partiellen Remissionen zwischen $4 \%$ und $9 \%$. Etwas erfolgversprechender war hingegen die Therapie bei gleichzeitiger Gabe von autologen tumorinfiltrierenden Lymphozyten, mit denen eine komplette Remissionsrate von $17 \%$ erzielt werden konnte. Fieber, Erytheme, Übelkeit sowie Erbrechen gehören jedoch zu den häufigen Nebenwirkungen dieser Therapie. Inwieweit sich diese Resultate durch den Einsatz gentechnisch manipulierter tumorinfiltrierender Lymphozyten oder autologer Nierenkarzinome im Sin- 
ne einer Vakzination verbessern lassen, kann z.Z. noch nicht beantwortet werden.

Ebenso liegen zur Effektivität einer Vakzinationsbehandlung beim Nierenzellkarzinom, wie von $M$. Kriegmair (München) und S. Pomer (Heidelberg) dargestellt, gegenwärtig noch keine klinischen Daten vor.

Zusammenfassend wurden von $H$. Rübben (Essen) folgende Therapieempfehlungen zur Behandlung des fortgeschrittenen Nierenzellkarzinoms gegeben:

- beim metastasierten Nierenzellkarzinom sollte eine Nephrektomie nur bei symptomatischen Patienten bzw. bei großen Tumoren mit einem kleinen Metastasenvolumen zur Beseitigung einer evtl. Hormonproduktion des Tumors durchgeführt werden;

- ausgewählte Fälle von Lungenmetastasen bei Nierenzellkarzinomen sollten der operativen Behandlung zugeführt werden;

- zur Optimierung der Zytokintherapie von Nierenzellkarzinomen sind größere Multicenterstudien erforderlich, da die Frage, welches Zytokin in welcher Kombination verwandt werden sollte, z.Z. nicht beantwortet werden kann;

- eine adjuvante Therapie beim lokal fortgeschrittenen Nierenzellkarzinom scheint nach den Ergebnissen der Delta-P-Studie nicht sinnvoll zu sein. Ebenso muß die Effektivität einer Vakzinationstherapie beim Nierenzellkarzinom mittels Phase-3-Studien dokumentiert werden;

- ein Immunmonitoring zytokinbehandelter Patienten sollte nur vor dem Hintergrund wissenschaftlicher Fragestellungen, jedoch nicht routinemäßig vorgenommen werden.
Dr. rer. nat. Walburgis Brenner

Urologische Klinik und Poliklinik

Klinikum der Johannes Gutenberg-Universität Mainz

Langenbeckstr. 1

55131 Mainz 